\title{
Neuropatia auditiva: aspectos clínicos, diagnósticos e terapêuticos
}

\section{Auditory neuropathy: clinical, diagnostic and therapeutic aspects}

Palavras-chave: neuropatia auditiva, desordem auditiva, perda da sincronia neural.

Key words: auditory neuropathy, hearing disorder, neural disynchronization.
Resumo

A auditivo, que gera uma dessincronia na condução nervosa, provavelmente relacionada com alterações de mielinização dessas fibras. Clinicamente, há uma perda auditiva e alteração na discriminação vocal. As otoemissões acústicas se apresentam normais com BERA não responsivo. Pode haver correlação com algumas patologias que cursam com neuropatia periférica. Os autores fazem uma revisão da literatura e relatam um caso de uma paciente portadora de neuropatia auditiva associada à retinose pigmentar com discussão das possíveis etiologias.

\section{Summary}

\begin{abstract}
A uditory neuropathy consists of an exclusive aggression of the auditory nerve, that causes a desynchrony in the nervous conduction, probably related to myelinization alterations of these fibers. Clinically, there is hearing loss and abnormal vocal discrimination. Otoacoustic emissions are normal with non-responsive ABR. It may be associated with some diseases that happen with peripheral neuropathy. The authors reviewed the literature and present a case of a patient with auditory neuropathy associated with pigmentar retinosis, with discussion of possible etiologies.
\end{abstract}

${ }^{1}$ Professor Titular de Foniatria da Faculdade de Fonoaudiologia da Pontifícia Universidade Católica de São Paulo. Médico da DERDIC/PUC.

${ }^{2}$ Pós-Graduanda da Disciplina de Otorrinolaringologia do Hospital das Clínicas da Universidade de São Paulo. Médica Assistente da Clínica de Otorrinolaringologia do Hospital do Servidor Público Municipal de São Paulo.

${ }^{3}$ Médica Otorrinolaringologista da Clínica Otorrinos - Recife. Ex-Residente da Clínica de Otorrinolaringologia do Hospital do Servidor Público Municipal de São Paulo. 


\section{INTRODUÇÃO}

Em anos recentes surgiram publicações colocando em cheque a ideia, prevalente anteriormente, de que as queixas de surdez apontavam apenas para a presença de falhas cocleares. De fato essas publicações não são originais pois, já no século XIX, autores apresentavam indicações clínicas da existência de sintomas auditivos causados por doenças externas às estruturas estritamente sensoriais. Mais ainda, na primeira metade do século XX e nos anos 60, trabalhos de autores conceituados mostravam quadros clínicos auditivos que não deviam ser cocleares mas que poderiam ser confundidos com deficiência auditiva sensorial ${ }^{5,6,8,9,10}$.

No início dos anos 80, com aprimoramento das técnicas de estudo sobre a eletrofisiologia do nervo auditivo e principalmente com a descoberta das Emissões Otoacústicas (EOAs), feita por Kemp em 1978, ficou mais fácil a análise da fisiologia auditiva e a separação das lesões que atingem a cóclea das que incidem sobre os outros pontos do sistema auditivo.

\section{A NEUROPATIA AUDITIVA}

A neuropatia auditiva resume-se anatomicamente em um acometimento exclusivo do nervo auditivo, que gera o que parece ser uma perda da sincronia na condução nervosa, muito provavelmente relacionada com uma alteração da mielinização dessas fibras. A localização precisa da alteração não está definida e pode diferir nos diversos casos, mas deve estar nas células ciliadas internas, nas sinapses entre as células ciliadas internas e o $8^{\circ}$ par, no $8^{\circ}$ par propriamente dito, ou ainda, em várias dessas estruturas ${ }^{3}$

Provavelmente o termo neuropatia surgiu da frequente associação, nesses pacientes, dos sintomas auditivos com os de outras neuropatias, como a neuropatia hereditária de Charcot-Marie-Tooth tipo II, a ataxia de Friedreich, as neuropatias do nervo óptico, a neuropatia de Guillan Barré e as neuropatias periféricas acompanhando os quadros de uremia, diabetes, exposição a cisplatina e kernicterus. O acometimento auditivo pode preceder em muitos anos o aparecimento da neuropatia periférica ${ }^{12}$.

Clinicamente pode haver perda auditiva e alteração da discriminação vocal, que é, normalmente, a principal queixa desses pacientes. Quando há associação do quadro com neuropatias periféricas, há alterações motoras e da marcha, além de redução de reflexos e da sensibilidade tátil.

A audiometria tonal revela uma perda bilateral, simétrica ou não. A alteração dos limiares auditivos pode variar quanto ao grau, indo de leve a profunda; as freqüências mais afetadas podem dar um traçado plano, curvas ascendentes e descendentes. A discriminação vocal está, muitas vezes, desproporcionalmente mais baixa em relação ao limiar auditivo tonal e pode haver alteração dos reflexos estapedianos.

As EOAs são normais, não havendo, no entanto, sinais de diminuição da amplitude das respostas com o uso de um ruído contralateral, binaural ou ipislateral, o que indica uma alteração do efeito supressor das vias auditivas eferentes ${ }^{1}$. Há ausência ou alteração de respostas na audiometria de tronco cerebral (BERA) a partir da onda I, sugerindo comprometimento das porções laterais do nervo auditivo e diferenciando a neuropatia auditiva das afecções do tronco cerebral, onde a onda I está presente. É possível ver, durante a realização da BERA, um microfonismo coclear amplo, se estendendo até 4 a 6 ms, que pode ser confundido, a princípio, com atividade elétrica do tronco cerebral, a não ser pelo fato de ele não se alterar com a diminuição da intensidade, mas sim com a inversão da polaridade do estímulo dado, ou seja, com a mudança de um clique condensado para um clique rarefeito.

\section{APRESENTAÇÃO DE CASO CLÍNICO}

R.A.M.G., sexo feminino, 17 anos de idade, solteira, natural e procedente de S.P., portadora de Retinose Pigmentar diagnosticada aos 11 anos de idade que progrediu com rápida perda visual há aproximadamente 2 anos. Procurou o ambulatório de Zumbido da Clínica de Otorrinolaringologia do Hospital do Servidor Público Municipal de São Paulo, com queixa de zumbido bilateral, constante, agudo, de fraca intensidade e dificuldade de discriminação da fala humana de caráter progressivo desde há aproximadamente seis meses, principalmente ao falar ao telefone e em ambientes ruidosos. Negava distúrbios do equilíbrio, alterações de locomoção e qualquer outra queixa otorrinolaringológica. De antecedentes familiares referia uma irmã, dois anos mais nova, com Retinose Pigmentar de evolução lenta e sem queixas auditivas. O exame otorrinolaringológico era normal. Clinicamente testamos a audição da paciente, durante a consulta, através da apresentação de palavras aleatórias e do pedido para que ela repetisse cada palavra dada. Apesar do acometimento ocular tomamos o cuidado de não fornecer pistas visuais durante o teste, que foi realizado a princípio com a sala em silêncio e posteriormente com o uso de um ruído competitivo. A paciente apresentou grandes dificuldades na realização da prova somente após a introdução do ruído de fundo. O exame audiométrico, realizado 8 meses antes da consulta, mostrava limiares dentro da normalidade (Figura 1), SRT à direita de $10 \mathrm{~dB}$ e à esquerda de $20 \mathrm{~dB}$, discriminação de $92 \%$ bilateralmente, ausência de reflexos e curva timpanométrica sem alterações. Foi solicitada uma nova avaliação audiológica composta de exame audiométrico, de uma BERA e de 
EOA. Essa nova audiometria evidenciou perda auditiva tipo neurossensorial mais acentuada em graves, bilateralmente, moderada à esquerda e leve à direita (Figura 2). A discriminação à direita foi de $80 \%$ a $60 \mathrm{~dB}$ para monossílabos e à esquerda de $72 \%$ a 80 dB, com rebaixamento para 48\% em 80 dB, quando em dissílabos na orelha esquerda. À direita não foi testada a discriminação para dissílabos em função do valor inicial de $80 \%$ para monossílabos. SRT a $25 \mathrm{~dB}$ à direita e a $40 \mathrm{~dB}$ à esquerda. Reflexos estapedianos estavam ausentes. A BERA, feita com estímulos na frequência de 8 estímulos por segundo, com um espectro de 1000 a 4000 Hz, com cliques condensados e rarefeitos com fins de sensibilização do exame, mostrou ausência total de respostas desde a onda I bilateralmente, tendo sido repetido o exame, confirmando-se os resultados. Não notamos alargamento do microfonismo coclear no traçado da BERA. As emissões otoacústicas transientes e por produtos de distorção estavam presentes e consistentes bilateralmente, exceto por uma queda em 6 e $8 \mathrm{KHz}$ nas emissões por produto de distorção à esquerda.

Foi solicitada Ressonância Nuclear Magnética de Crânio que não apresentou alterações.

\section{DISCUSSÃO}

A neuropatia auditiva, até há pouco tempo desconhecida, tem sido, nos dias de hoje, cada vez mais estudada e diagnosticada. Sabe-se que o diagnóstico de Neuropatia Auditiva se faz através de uma ausência ou alteração importante na BERA desde a onda I, juntamente com a presença de EOA normais. Sabe-se também que, de uma forma geral, nesses pacientes, o reflexo do estapédio encontra-se alterado e que, invariavelmente a dificuldade de discriminação vocal é uma das queixas mais importantes ${ }^{12}$. Todas essas características são encontradas no caso apresentado. Além disso, os pacientes com neuropatia auditiva possuem uma alteração do efeito supressor das emissões otoacústicas provocado pelas vias auditivas eferentes, teste esse que infelizmente não pudemos realizar.

A avaliação clínica da audição, feita durante a consulta, apesar de não ser baseada em fatores rígidos, nem tampouco fornecer dados quantitativos, deu-nos uma idéia de como estava alterada a audição e a discriminação da paciente, principalmente na presença de ruído competitivo, apesar da audiometria trazida estar normal.

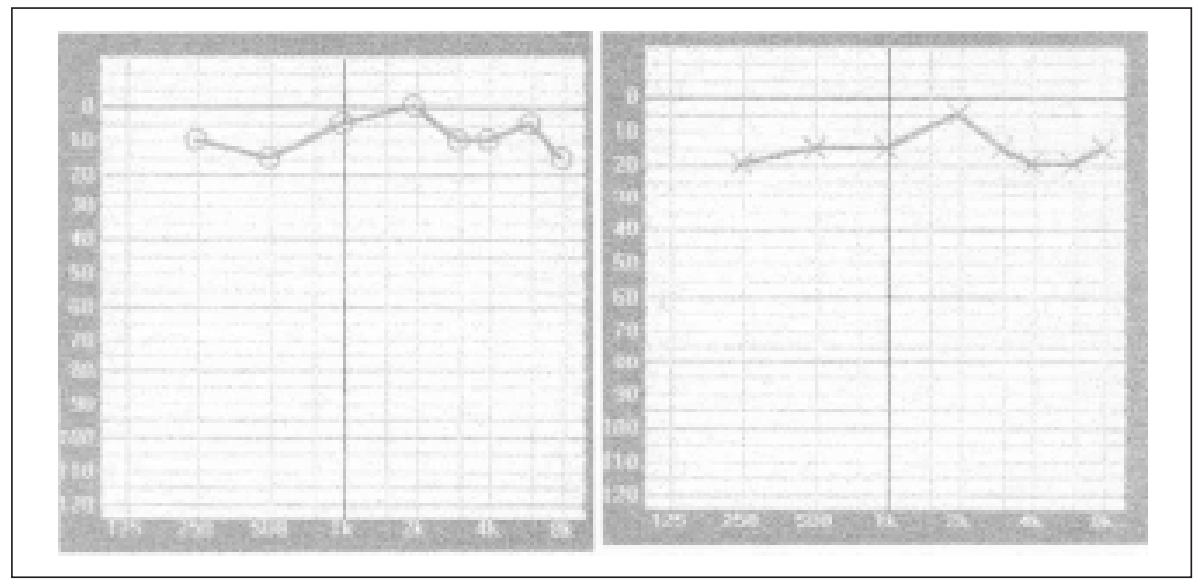

Figura 1. Audiometria realizada antes da primeira consulta.

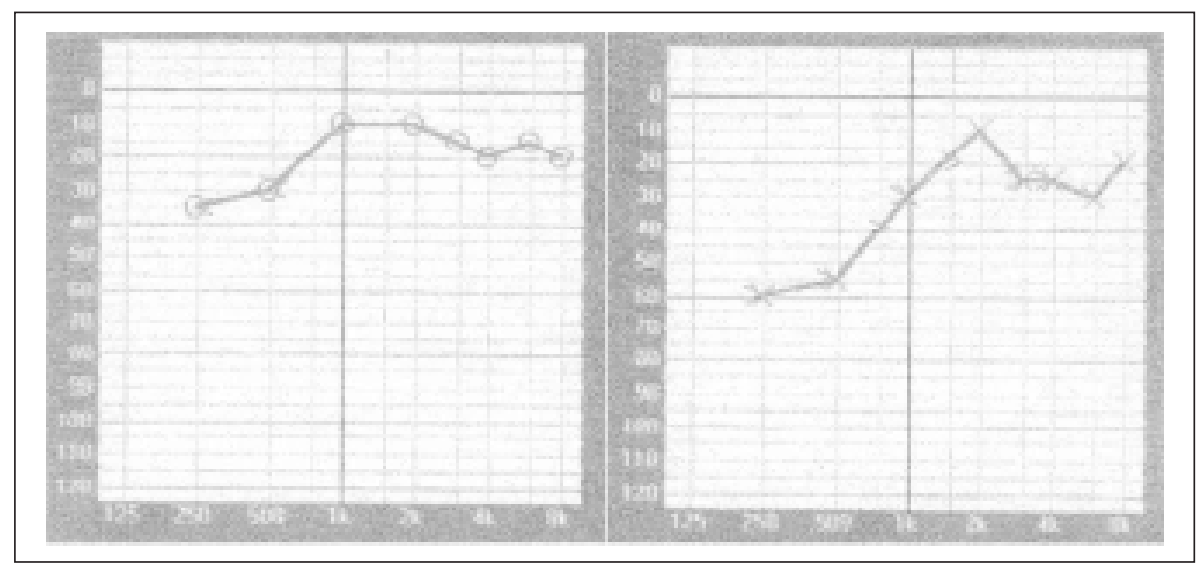

Figura 2. Audiometria realizada após a primeira consulta. 
A nova audiometria mostrou uma rápida evolução do padrão audiométrico, com elevação dos limiares, principalmente em graves, perda assimétrica, maior à esquerda, e uma piora da porcentagem de discriminação vocal quando se passou do teste com monossílabos para o teste com dissílabos, indicando que a redundância, que é um fator facilitador na perda coclear, na neuropatia auditiva leva a uma piora no reconhecimento. Isso ocorre devido a perda do sincronismo neural das fibras auditivas que acarreta uma severa alteração na habilidade do processamento temporal prejudicando muito a discriminação da fala humana. ${ }^{14}$

Pacientes com neuropatia auditiva podem apresentar um microfonismo longo que, durante a realização da BERA, pode ser confundido com uma possível resposta do nervo auditivo. A inversão da polaridade do estímulo, por exemplo, a passagem de um estímulo condensado para um rarefeito, acaba invertendo também a polaridade das ondas se essas forem produto do microfonismo coclear, mas não altera a polaridade se for resposta do nervo auditivo. Esse método, de fácil realização, deve ser sempre usado como forma de sensibilização do exame e detecção de respostas mais confiáveis. No caso apresentado não identificamos esse alargamento do microfonismo coclear.

Da mesma forma que o microfonismo coclear, as emissões otoacústicas também são produto das células ciliadas externas e por esse motivo encontram-se presentes e normais.

A Ressonância Nuclear Magnética foi pedida para descartar algum acometimento em tronco cerebral que pudesse estar causando essa perda auditiva, já que havia uma perda com características retrococleares. Como seria de se esperar na neuropatia auditiva, o exame não apresentou alterações.

A presença da Retinose Pigmentar, diagnosticada 6 anos antes do início dos sintomas auditivos e que nos últimos 2 anos evoluiu de forma muito rápida, com perda importante da acuidade visual é um fator agravante nessa paciente, já que aumenta a privação sensorial e modifica a terapia convencional, requerendo um maior treinamento da sensibilidade tátil.

Há cerca de nove tipos de síndromes hereditárias, a maioria de transmissão autossômica recessiva, que acometem o sistema auditivo e visual nos seus mais diversos pontos, levando às mais diversas combinações de perda auditiva e deficiência visual? .

A associação mais frequente de retinose pigmentar e deficiência auditiva é encontrada na Síndrome de Usher, uma doença autossômica recessiva, que é classificada em quatro tipos, dependendo das características da perda auditiva, do acometimento vestibular ou não, e da época de início dos sintomas ${ }^{11}$. De uma forma geral, pela análise histológica de ossos temporais desses pacientes, sabe-se que o gânglio espiral pode estar envolvido em alguns casos, mas há um acometimento das células ciliadas externas de forma muito frequente ${ }^{13}$.

A neuropatia auditiva é, em muitos casos, descrita na literatura, acompanhando quadros de ataxias hereditárias e de neuropatias periféricas como, por exemplo, as Síndromes de Refsum, de Charcot-MarieTooth, a Ataxia de Friederich, a Síndrome de Guillan-Barré, sendo que em todas essas pode ocorrer o acometimento óptico concomitante ${ }^{2}$. Esses pacientes com neuropatias periféricas não-auditivas podem apresentar alterações dos reflexos, alterações motoras e sensitivas, alterações de marcha, movimentos coreicos, presença de nistagmos e vertigem ${ }^{4}$.

Sabe-se, também, que o acometimento auditivo e ocular pode preceder as outras neuropatias, com ou sem distúrbios do equilíbrio e marcha, em muitos anos. Bogaert \& Martin $(1974)^{2}$, em uma extensa revisão, citam um relato de 1924 de uma paciente com retinose pigmentosa, que com 10 anos iniciou um quadro de surdez progressiva e que, com 34 anos, desenvolveu ataxia cerebelar. Starr e colaboradores $^{12}$ ao descreverem seus pacientes, com neuropatia auditiva e periférica não-auditiva, afirmam que dois deles, com idade de 4 e 16 anos, os mais jovens, só apresentavam o acometimento auditivo, mas que muito provavelmente o quadro periférico apareceria com o passar dos anos. Essas afirmações nos levam a interrogar se a nossa paciente não virá a desenvolver um quadro semelhante, apesar de ainda não haver nenhum sinal clínico que indique isso.

Até esse momento, não tivemos condição de diagnosticar nenhuma das síndromes citadas como causadora dos sintomas observados, possivelmente por faltarem dados a serem obtidos em avaliações neurológicas, otoneurológica, eletromiográfica e genética, que ainda não foram realizadas por razões pessoais da paciente, não estando, portanto, descartada, a presença de neuropatia periférica extra-auditiva incipiente, na paciente e na sua irmã mais nova, portadora de retinose pigmentar.

\section{COMENTÁRIOS FINAIS}

O diagnóstico de neuropatia tende agora a ser feito com maior freqüência, o que certamente trará benefícios a inúmeros indivíduos que anteriormente eram atendidos equivocadamente, mas, ainda estamos distantes de generalizar os conhecimentos e esclarecer as formas de diagnóstico mais completas de patologias auditivas não cocleares.

Tais conhecimentos, formas e diagnósticos envolvem desde a procura de prevalências relativas a cada uma - neuropatia e patologias centrais - até a elaboração de sistemáticas diagnósticas que contenham a participação de dados otológicos, neurológicos e foniátricos, devido às variáveis presentes nesse campo. 
É inegável que ao lado de incertezas e dificuldades, o acúmulo de conhecimento trazidos por estudos de casos e refinamentos na avaliação das respostas auditivas comportamentais e eletrofisiológicas, de detecção de emissões da cóclea, deverão tornar cada vez mais distante o tempo de diagnóstico equivocados, imprecisos, em pessoas com sintomas auditivos estranhos, com respostas incongruentes nas diversas formas de avaliação.

\section{REFERÊNCIAS BIBLIOGRÁFICAS}

1. BERLIN, C.I. - Auditory Neuropathy. Using OEAs and ABR from Screening to Management. Seminars in Hearing, 20 (4): 307-15, 1999.

2. BOGAERT, L.V.; MARTIN, L. - Optic and cochleovestibular degenerations in the hereditary ataxias. Brain. 97:15-40, 1974.

3. DOYLE, K.J.; SININGER, Y.; STARR, A. - Auditory Neuropaty in Childhood. Laryngoscope 108:1374-7, 1998.

4. CASSANDRO, E.; MOSCA, F.; SEQUINO, L.D.E; FALCO, F.A.; CAMPANELLA, G. - Otoneurological finding in Friedreich's ataxia and other inherited neuropathies. Audiology. 25(2):84-91, 1986.

5. GREINER, C. - Alterações Neurógenas e Centrais. In: BERENDES, J; LINK, R.; ZOLLNER, F. - Tratado de Otorrinolaringologia. Barcelona, Editorial Científico Médica, 1970.
6. HALLPIKE, C.S.; HARRIMAN, D.G.F.; WELLS, C.E.C.- A Case of Afferent Neuropathy and Deafness. J. Laryngol Otol 94:945-64, 1980.

7. KONIGSMARK, B.W. - Hereditary deafness in man. N. England J.Medicine. 14:774-8, 1969

8. LUCHISINGER, R; ARNOLD, G.E. - Voice-Speech-Language. London, Constable, 528-43, 1965.

9. MYKLEBUST, H.R. - Auditory Disorders in Children. New York, Grune \& Stratton, 1954.

10. QUIRÓS, J.B. DE - Las Lhamadas Afasias Infantiles. Buenos Aires, C.E.D.I.F.A., 69-94, 1971

11. SHINKAWA, H.; NADOL, J.B. - Histopathology of inner ear in usher's syndrome as observed by light and electron microscopy.Ann Otol Rhinol Laryngol. 95:313-7, 1986.

12. STARR, A.; PICTON, T.W.; SININGER, Y.; HOOD, L.J.; BERLIN, C. Auditory Neuropathy. Brain 119:741-53, 1996.

13. WAGENAAR, M.; SCHUKNECHT, H.; NADOL, J.; RENS, M. B.; DAHL, S.P.; KIMBERLING, J.; CREMERS, C. - Histopathologic Features of the temporal bone in Usher Syndrome Type I. Arch Otolaryngol Head Neck Surg. 126:1018-23, 2000.

14. ZENG, F.G.; OBA, S.; GARDE, S.; SININGER, Y.; STARR, A. - Temporal and speech processing deficits in auditory neuropathy. Neuroreport. 10(16):3429-35, 1999. 